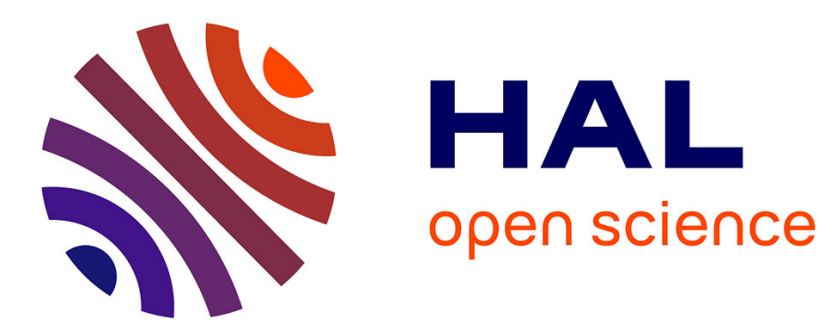

\title{
Dépassé, le patriarcat ?
}

Nicole Mosconi, Marion Paoletti

\section{To cite this version:}

Nicole Mosconi, Marion Paoletti. Dépassé, le patriarcat ?. Travail, genre et sociétés, 2017, La gestation pour autrui en débat, 38, pp.171-174. 10.3917/tgs.038.0171 . halshs-01643236

\section{HAL Id: halshs-01643236 \\ https://shs.hal.science/halshs-01643236}

Submitted on 24 May 2019

HAL is a multi-disciplinary open access archive for the deposit and dissemination of scientific research documents, whether they are published or not. The documents may come from teaching and research institutions in France or abroad, or from public or private research centers.
L'archive ouverte pluridisciplinaire HAL, est destinée au dépôt et à la diffusion de documents scientifiques de niveau recherche, publiés ou non, émanant des établissements d'enseignement et de recherche français ou étrangers, des laboratoires publics ou privés.

\section{(이) $\$$}

Distributed under a Creative Commons Attribution - NonCommercial - NoDerivatives| 4.0 


\section{DÉPASSÉ, LE PATRIARCAT ?}

Nicole Mosconi et Marion Paoletti

\section{La Découverte | «Travail, genre et sociétés »}

$2017 / 2 \mathrm{n}^{\circ} 38$ | pages 171 à 174

ISSN 1294-6303

ISBN 9782707197511

\section{Article disponible en ligne à l'adresse :}

https://www.cairn.info/revue-travail-genre-et-societes-2017-2-page-171.htm

Distribution électronique Cairn.info pour La Découverte.

(C) La Découverte. Tous droits réservés pour tous pays.

La reproduction ou représentation de cet article, notamment par photocopie, n'est autorisée que dans les limites des conditions générales d'utilisation du site ou, le cas échéant, des conditions générales de la licence souscrite par votre établissement. Toute autre reproduction ou représentation, en tout ou partie, sous quelque forme et de quelque manière que ce soit, est interdite sauf accord préalable et écrit de l'éditeur, en dehors des cas prévus par la législation en vigueur en France. Il est précisé que son stockage dans une base de données est également interdit. 

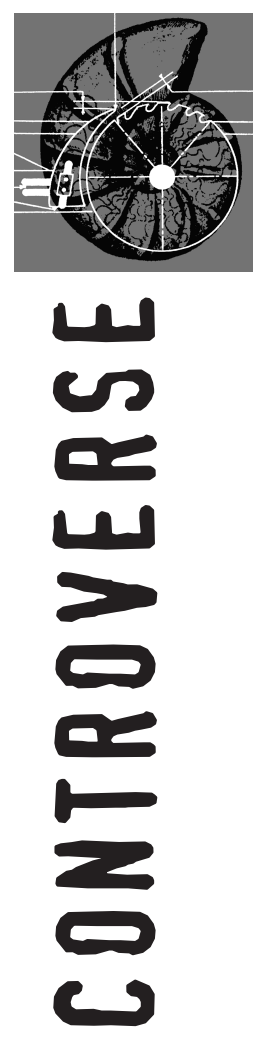

\section{DÉPASSÉ,} LE PATRIARCAT ?

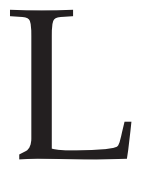

a décennie 1970 a constitué un moment de changements politiques, culturels et épistémologiques profonds et durables parce que les mouvements féministes ont interrogé la domination patriarcale. Ce faisant, la problématisation de la séparation entre sphère publique et sphère privée a permis de penser la domination masculine dans la famille, la conjugalité comme un problème inhérent à l'ordre politique et de constituer de nouveaux objets d'études tels que la division sexuelle du travail et l'appropriation du travail gratuit des femmes au sein du foyer, alors minutieusement décrites [Parini, 2013]. L'analyse en termes de patriarcat a été centrale pour comprendre la domination masculine et pour élargir les possibilités de penser et d'agir [Delphy, 1998 ; Guillaumin, 1992 ; Mathieu, 1991].

En France, dans les années 1970, c'est d'abord une matrice structuraliste et matérialiste qui permet de concevoir le genre comme " un rapport social de sexe », une matrice déterminante dans la conceptualisation du patriarcat pour penser la domination masculine. Les femmes en tant que groupe social partagent une situation commune d'oppression ou de domination masculine, matérielle et culturelle, dont les fondements résident dans le système de parenté et le mariage : la notion de patriarcat rend compte de ces analyses. La domination se distingue de la relation de pouvoir par sa durée et sa forme instituée [Laufer, 2005]. La dénonciation 
politique du patriarcat a permis une démocratisation conjugale et sexuelle [Singly 1996 ; Fassin, 2005]. Or cette approche structuraliste, en grande partie axée sur la reproduction, la reconduction et le maintien des inégalités, bute sur la question du changement, en particulier dans les sociétés occidentales depuis les années 1970. Si le patriarcat trouve sa source avant tout dans les familles et le couple, les changements sociaux intervenus depuis les années 1970 doivent-ils amener à considérer le patriarcat comme une situation et un concept dépassés?

L'approche structuraliste a été critiquée à partir des années 1980 en France en raison de cette difficulté intrinsèque à penser le changement, mais aussi pour son absence d'historicisation, sa prétention à l'universalité et parce qu'elle laisse peu de places aux acteurs et actrices ainsi qu'à leur subjectivité. Ces critiques générales en sciences sociales dans la décennie 1980 ont poussé les études de genre vers le point de vue alternatif de Michel Foucault sur le pouvoir, déplaçant l'attention des structures qui produisent des groupes sociaux vers les modes d'action des individus. Contre une approche matérialiste des faits sociaux, des analyses focalisées sur le langage, les individus et les relations de pouvoir se sont développées, notamment à la faveur de la diffusion des travaux de Joan Scott. Réversible, fluide, fragmenté, le pouvoir n'empêche cependant pas de penser la discipline, comme soumission des acteurs et actrices aux normes de genre, mais en tenant compte de leur subjectivité. La rupture épistémologique qu'a constituée la définition du genre comme relation de pouvoir a permis le développement de travaux qui ont souvent abandonné, à partir des années 1980-1990, le patriarcat comme catégorie centrale des études féministes, sans que cet abandon (relatif) soit pour autant discuté. Le changement de paradigme (ou plutôt leur hybridation) a progressivement effacé le terme même dans les études de genre en France. Doit-on aller jusqu'à abandonner la notion de patriarcat?

C'est à une telle discussion qu'ouvre l'essai d'Éric Macé, paru en 2015 et intitulé L'après-patriarcat. Par la prise de position qui est celle de l'auteur, le débat se prête particulièrement bien à controverse. Explicitement située dans une sociologie des rapports de pouvoir qui s'oppose à la sociologie de la domination, la perspective d'Éric Macé, que l'auteur présente ici, invite à prendre en compte les effets transformateurs des mouvements féministes, homosexuels, queer et trans, afin de relativiser le caractère heuristique de la notion de patriarcat pour décrire aujourd'hui, ici et ailleurs, les rapports de genre. "Les arrangements de genre contemporains » doivent pour l'auteur être définis comme " post patriarcaux » au sens où « la mise en asymétrie du masculin et du féminin, bien que persistante dans des pratiques et des 
représentations, n'est plus ni légitime ni nécessaire » [Macé, 2015, p. 25].

Cette position suscite un certain nombre de réserves, exprimées ici par Clément Arambourou dans une discussion fine et serrée du texte d'Éric Macé. Les principales retenues sont sans doute que "l'historicisation », nécessaire, « du patriarcat n'appelle pas forcément de rupture avec une pensée de la domination » et que " parler de domination en sciences sociales ne conduit pas à nier l'existence et l'autonomie des rapports de pouvoir inter-individuels ». L'hypothèse du post patriarcat, qui demande à être vérifiée, ne doit pas conduire, dans le même geste, à abandonner la boîte à outils théorique du marxisme, toujours utile pour les études de genre. Il se pourrait, pour reprendre les termes de Françoise Thébaud, que le post patriarcat soit " plus politique et culturel que sociologique ». [2003, p. 79]

Au-delà de cette discussion théorique, nous avons voulu proposer à des spécialistes de l'Afrique et de l'Inde d'examiner les vertus possibles de la notion de "post patriarcat » pour évaluer les changements dans des sociétés différentes. Pour l'Afrique, Madeleine Wayack Pambé et Nathalie Sawadogo montrent que la notion de patriarcat, considéré comme une importation occidentale pour expliquer les rapports de sexe dans leur société, constitue un point de clivage majeur au sein des féminismes africains. Cependant la notion de "patriarcat modernisé » proposée par Éric Macé leur apparaît utile pour décrire les évolutions contrastées et contradictoires des sociétés africaines vers davantage d'égalité entre les femmes et les hommes. Elle vise alors à décrire " une coexistence des rapports de sexe traditionnels avec ceux hérités de la modernité occidentale conduisant à des identités et pratiques de genre composites ».

Quant à l'Inde, Kamala Marius considère que, quelles que soient les évolutions législatives vers une égalité formelle, parler de "post patriarcat» est peu utile. La notion de patriarcat conserve toute sa pertinence pour décrire une société marquée par la prééminence de l'homme au sein de la famille et de la relation conjugale, encore considérée généralement comme légitime et nécessaire. Il faut néanmoins prendre en compte la complexité qu'impliquent le contexte postcolonial et les divisions de la société indienne selon les castes, classes, races, religions, sexes, etc.

La discussion empirique à partir de sociétés différentes signale combien la notion de post patriarcat peut être prise pour une hypothèse, stimulante certes mais controversée, demandant à être testée dans les faits et appelant un « horizon de recherches à venir » [Neveu, 2016]. 


\section{BIBLIOGRAPHIE}

Delphy Christine, 1998, L'ennemi principal. Tome 1: Économie politique du patriarcat, Paris, Syllepse.

FASSIN Éric, 2005, «La démocratie sexuelle et l'intellectuel démocratique », introduction à L'Inversion de la question homosexuelle, Paris, éd. Amsterdam.

Guillaumin Colette, 1992, Sexe, race et pratique du pouvoir. L'idée de nature, Paris, Côté-Femmes.

LaUfer Jacqueline, 2005, "Domination », in Margaret Maruani (dir.) Femmes, genre et sociétés : L'état des savoirs. Paris, La Découverte, pp. 67-75.

MAcÉ Éric, 2015, L'après-patriarcat, Paris, Seuil.

Mathieu Nicole-Claude, 1991, L'Anatomie politique. Catégorisations et idéologies du sexe, Paris, Côté-Femmes.

Neveu Erik, 2016, "Compte-rendu de : Macé (Éric), L'après-patriarcat », Revue française de science politique, vol. 66, $\mathrm{n}^{\circ} 2$, pp. 340-342.

PArINI Lorena, 2013, "Domination/Pouvoir », in Dictionnaire. Genre et science politique, Paris, Presses de Sciences Po (PFNSP), « Références », pp. 180-190.

SingLY (de) François, 1996, Le Soi, le couple et la famille, Paris, éd. Nathan.

THÉBAud Françoise, 2003, Écrire L'histoire Des Femmes, Lyon, ens Éditions. 